\title{
A Completeness theorem for Formal Topologies
}

\author{
Silvio Valentini \\ Dipartimento di Matematica Pura ed Applicata \\ Università di Padova \\ via G. Belzoni n.7, I-35131 Padova, Italy \\ email: valentini@pdmat1.math.unipad.it
}

September 24, 1996

\begin{abstract}
The main mathematical result of this work is a quite simple formulation and proof of a Rasiowa-Sikorski-like theorem for countable lattices. Then the paper suggests an interpretation of this mathematical result as a completeness theorem for the formal topologies introduced by G. Sambin in order to provide a constructive approach to topology which is expressible within Martin Löf's intuitionistic theory of types. This completeness theorem shows that, as long as one is interested in dealing only with the coverage relation between two open sets of a topology, within a constructive framework, a very simple mathematical structure is needed. It is necessary to stress that the completeness theorem holds because of the "weak" intuitionistic set and subset theory that we use when dealing with formal topologies.
\end{abstract}

\section{Introduction}

In this paper we will show a completeness theorem for formal topologies [Sambin 87, Sambin 88], which we are briefly going to recall in the next section. It shows that, as long as one is interested in dealing only with the coverage relation between two open sets of a topology, in an intuitionistic framework, a very simple mathematical structure is needed.

Since formal topologies are intended to be a constructive approach to the classical, point-set topology, the first obliged step to define them is to introduce a theory of subsets which may be intuitionistically acceptable. To this purpose we will use a suitable extension of Martin-Löf's Intuitionistic Theory of Types (briefly ITT in the following) [Martin-Löf 84], which is described in full extent in [Sambin-Valentini 9?]; here we will recall the definitions that we need at the beginning of the next section.

Then we will classically prove a Rasiowa-Sikorski-like theorem for the countable lattices which shows that it is possible to find a prime filter which respects 
a countable collection of suprema, in the same way the original theorem by Rasiowa and Sikorski does to prove the completeness theorem for first order logic [Rasiowa-Sikorski 51]. Such a theorem is the key to embed any countable distributive lattice into a point-set topology. It will be used in the last section to prove the wished completeness theorem for the formal topologies.

\section{Formal topologies}

In order to develop a constructive version of the classical topology we will need to deal with sets and subsets in an intuitionistic framework. While for the set theory we commit ourselves to Martin-Löf's Intuitionistic Theory of Types [Martin-Löf 84], in order to deal with subsets a specific notion for subsets must be adopted, together with some notation. Here we will illustrate only the main definitions that we are going to use, while for a deeper treatment the reader is referred to [Sambin-Valentini 9?].

The distinction between sets and collections ${ }^{1}$ is basic in ITT; a collection is a set only if one can effectively produce its elements. For instance $\mathbf{N}$, i.e. natural numbers, is a set since its elements are (equivalent to) 0 or the successor of any element already known to be in $\mathbf{N}$, whereas, in general, the collection of all the classical subsets of a set $S$ cannot be a set insomuch as one cannot produce all of its elements. ${ }^{2}$

In general not only is the collection of all the subsets of a set not a set, but even one subset of a set can be only a collection but no set; as an example, consider the subset of $\mathbf{N}$ of the code numbers of the recursive functions which do not halt on 0: because of the unsolvability of the "halting problem", there is no way to effectively produce all the elements of this subset. On the other hand, being able to deal with subsets is necessary to develop almost any piece of mathematics. For this reason, we introduce the following notion of subset, which is suggested by the axiom of separation of ZF set theory. Let $S$ be a set; then we put

$$
U \subset S \equiv(x: S) U(x) \text { prop }
$$

that is, we say that $U$ is a subset of $S$ whenever $U$ is a propositional function on elements of $S$. In what follows also the alternative, usual, notation $\{x \in S$ : $U(x)$ true $\}$ will be used for the subset $U$ of $S$, in order to make the exposition clearer. ${ }^{3}$

The subset-theory which results from this definition is a sort of local set theory, since all the relations and operations we introduce are always relativized

\footnotetext{
${ }^{1}$ In this paper we systematically use the word "collection" for what is called "category" in ITT [Martin-Löf 84].

${ }^{2}$ As a further example, consider that one can form the function space $\mathbf{N} \rightarrow \mathbf{N}$ in ITT but the type he obtains corresponds in no way to the collection of all the classical functions from $\mathbf{N}$ to $\mathbf{N}$.

${ }^{3}$ The alternative possibility to identify the subset of the elements of $S$ which satisfy the proposition $U(x)$ prop $[x: S]$ with the ITT type $\Sigma(S, U)$ compels to define asU by "there is an element b such that $\langle a, b\rangle \in \Sigma(S, U)$ " and hence $a \varepsilon U$ cannot be a ITT proposition.
} 
to a set; to make this fact explicit, we will indicate the set as an index (even if in the following we will sometimes omit it when it is clear from the context).

The first definition is membership; this definition is an immediate consequence of the fact that $U$ is a propositional function:

$a \varepsilon_{S} U$ true $\equiv a \in S$ and $U(a)$ true, i.e. there is a proof of the proposition $U(a)$

The next step is the definition of inclusion between subsets of $S$, which is an obvious consequence of the previous definition of membership:

$$
U \subseteq S V \equiv(\forall x \in S)(U(x) \rightarrow V(x))
$$

which in turn gives

$$
U={ }_{S} V \equiv(\forall x \in S)(U(x) \leftrightarrow V(x))
$$

As usual, given a proposition over elements of $S$, i.e. $A(x)$ prop $[x: S]$, universal quantification over a subset $U \subset S$, i.e. $\left(\forall x \varepsilon_{S} U\right) A(x)$, is nothing but an abbreviation for $(\forall x \in S)(U(x) \rightarrow A(x))$; similarly $\left(\exists x \varepsilon_{S} U\right) A(x)$ is just an abbreviation for $(\exists x \in S)(U(x) \& A(x))$.

Then subset operations can be introduced: in this paper we only need binary intersection and union and arbitrary union. Given two subsets $U, V$ of $S$ and a family $\left(V_{i}\right)_{i \in I}$ of subsets of $S$, i.e. a propositional function $V(i, x)$ prop $[i$ : $I, x: S]$, we put:

$$
\begin{aligned}
& U \cap_{S} V \equiv(x: S) U(x) \& V(x) \text { prop } \\
& U \cup_{S} V \equiv(x: S) U(x) \vee V(x) \text { prop } \\
& \cup_{i \in I} V_{i} \equiv(x: S)(\exists i \in I) V(i, x) \text { prop }
\end{aligned}
$$

It should be clear that, given any set $S$ and a fixed countable language, as is the case for ITT, the number of "subsets" of such a set is at most countable since only a countable collection of propositional functions on its elements can be formed. While in general this fact gives rise to a weak set theory, in our approach it is an essential feature to prove the completeness theorem: using a little slogan we can say that "we win because of our weakness".

Let us now turn to formal topologies. Formal topologies were introduced by G. Sambin as a constructive approach to topology [Sambin 87], in the tradition of the Johnstone's version of the Grothendieck topologies [Johnstone 82] and Fourman and Grayson's Formal Spaces [Fourman-Grayson 82], but using simpler technical devices and a constructive set theory based on Martin Löf's Intuitionistic Theory of Types. Today, formal topologies are also a well developed device to present locales and to study their properties [Battilotti-Sambin 94].

The main point in the formal approach to topology is the study, in a constructive framework, of the properties of a topological space $\langle X, \Omega(X)\rangle$, where $\Omega(X)$ is the family of the open subsets of the collection $X$, which can be expressed without any reference to the points, that is to the elements of the collection $X$.

Since a point-set topology can always be presented using one of its bases, the abstract structure that we will consider is a commutative monoid $\left\langle S, \bullet \mathcal{S}, 1_{\mathcal{S}}\right\rangle$ 
where the set $S$ corresponds to the set of the elements of the base of the point-set topology $\Omega(X), \bullet_{\mathcal{S}}$ corresponds to the operation of intersection between basic elements, and $1_{\mathcal{S}}$ corresponds to the whole collection $X$.

In a point-set topology any open set is obtained as a union of elements of the base, but union does not make sense if we refuse any reference to points; hence we are naturally lead to think that an open set may directly correspond to a subset of the set $S$. Unfortunately this idea is not completely correct since there may be many different subsets of basic elements whose union is the same open set; hence, in order to better define what corresponds to an open set, we need to introduce also an equivalence relation $\cong_{\mathcal{S}}$ between two subsets $U$ and $V$ of $S$ such that $U \cong_{\mathcal{S}} V$ holds if and only if, denoting by $c^{*}$ the element of the base which corresponds to the formal basic open $c$, the opens $U^{*} \equiv \cup_{a \varepsilon U} a^{*}$ and $V^{*} \equiv \cup_{b \varepsilon V} b^{*}$ are equal. To this purpose we introduce an infinitary relation $\triangleleft_{\mathcal{S}}$, which will be called cover, between a basic element $a$ of $S$ and a subset $U$ of $S$ whose intended meaning is that $a \triangleleft_{\mathcal{S}} U$ when $a^{*} \subseteq U^{*}$. The conditions we will require of this relation are a straightforward rephrasing of the similar set-theoretic situation. It will be the aim of the completeness theorem to show that they are also sufficient.

Definition 2.1 (Formal topology) Let $S$ be a set, then a formal topology over $S$ is a structure

$$
\mathcal{S} \equiv\left\langle S, \bullet \mathcal{S}, 1_{\mathcal{S}}, \triangleleft_{\mathcal{S}}\right\rangle
$$

where $\bullet_{\mathcal{S}}$ is an associative and commutative operation over $S, 1_{\mathcal{S}}$ is the unit of the operation $\bullet_{\mathcal{S}}$ and $\triangleleft_{\mathcal{S}}$ is an infinitary relation between an element of $S$ and a subset of $S$ such that, for any $a, b \in S$ and for any $U, V \subset S$ the following conditions hold

$$
\begin{aligned}
& \text { (reflexivity) } \frac{a \varepsilon_{S} U}{a \triangleleft_{\mathcal{S}} U} \\
& \text { (transitivity) } \frac{a \triangleleft_{\mathcal{S}} U \quad\left(\forall u \varepsilon_{S} U\right) u \triangleleft_{\mathcal{S}} V}{a \triangleleft_{\mathcal{S}} V} \\
& \text { (•-Left) } \\
& (\bullet-R i g h t) \\
& \frac{a \triangleleft_{\mathcal{S}} U}{a \bullet b \triangleleft_{\mathcal{S}} U} \\
& \frac{a \triangleleft_{\mathcal{S}} U \quad a \triangleleft_{\mathcal{S}} V}{a \triangleleft_{\mathcal{S}}\left\{u \bullet v: u \varepsilon_{S} U, v \varepsilon_{S} V\right\}}
\end{aligned}
$$

Let us introduce two abbreviations which will make the notation easier:

$$
\begin{aligned}
U \triangleleft_{\mathcal{S}} V & \equiv\left(\forall u \varepsilon_{\mathcal{S}} U\right) u \triangleleft_{\mathcal{S}} V \\
U \bullet V & \equiv\left\{u \bullet v: u \varepsilon_{\mathcal{S}} U, v \varepsilon_{\mathcal{S}} V\right\}
\end{aligned}
$$

These abbreviations allow a much more compact writing of the conditions above; besides the following derived conditions appear clearer by far.

$$
\begin{array}{cc}
\text { (global reflexivity) } & U \triangleleft_{\mathcal{S}} U \\
\text { (global transitivity) } & \frac{U \triangleleft_{\mathcal{S}} V}{U \triangleleft_{\mathcal{S}} W}
\end{array}
$$

Moreover they suggest a way to define the relation $\cong_{\mathcal{S}}$ that we were looking for. In fact by putting 


$$
U \cong_{\mathcal{S}} V \equiv U \triangleleft_{\mathcal{S}} V \text { and } V \triangleleft_{\mathcal{S}} U
$$

we obtain an equivalence relation between subsets of $S$ whose intended meaning is just that for any $u \varepsilon U, u^{*} \subseteq V^{*}$ and for any $v \varepsilon V, v^{*} \subseteq U^{*}$, which shows that $U^{*}=V^{*}$. We can rephrase somewhat more formally what we have achieved by identifying an open set with the equivalence class induced by the equivalence relation $\cong_{\mathcal{S}}$, i.e. we will call $[U] \equiv\left\{V: U \cong \cong_{\mathcal{S}} V\right\}$ a formal open of the formal topology $\mathcal{S}$.

We can bring a countable distributive lattice $\operatorname{Open}(\mathcal{S})$, actually a Heyting algebra, out of the collection of the formal opens of the formal topology $\mathcal{S}$ by putting:

$$
\begin{array}{rlc}
0_{O \operatorname{Pen}(\mathcal{S})} & \equiv & {[\emptyset]} \\
1_{\operatorname{Open}(\mathcal{S})} & \equiv & {[S]} \\
{[U] \wedge_{O \operatorname{Pen}(\mathcal{S})}[V]} & \equiv & {[U \bullet \mathcal{S} V]} \\
{[U] \vee_{O \operatorname{Pen}(\mathcal{S})}[V]} & \equiv & {[U \cup V]}
\end{array}
$$

and we can extend the definition to the suprema over any indexed family of subsets by putting

$$
\vee_{i \in I}\left[V_{i}\right] \equiv\left[\cup_{i \in I} V_{i}\right]
$$

It is not difficult to check that $\operatorname{Open}(\mathcal{S})$ is indeed a lattice which is distributive even with respect to suprema over any indexed family of subsets since an implication operation between formal opens can be defined by putting

$$
[U] \rightarrow_{O p e n(\mathcal{S})}[V] \equiv\left[\left\{w \in S:\{w\} \bullet_{\mathcal{S}} U \triangleleft_{\mathcal{S}} V\right\}\right] .
$$

Moreover the lattice $\operatorname{Open}(\mathcal{S})$ is countable since it has at most as many elements as the propositional functions on elements of $S$ are.

It should be clear now the reason why we call our topologies formal: the open sets are in fact purely formal objects since they are not "made of points". So, proving the completeness theorem means showing that any formal open can be filled with points in order to obtain a point-set standard topology, i.e. to show that the formal topologies have enough points ${ }^{4}$. The idea, borrowed from the usual Stone representation theorems, is to look for the points among the prime filters of $\operatorname{Open}(\mathcal{S})$. In fact let us recall how formal points can be introduced in formal topology (see [Sambin 87]).

We start by looking for the conditions a point must satisfy which are expressible in the language of the formal topologies; to this aim, supposing $\mathcal{S}$ to be a formal topology and $a$ one of its basic open subset, let us write $x \Vdash a$ to mean that the point $x$ belongs to the basic open $a$. The first obvious condition is that any point belongs to the whole topological space and it can be stated by requiring that for any point $x$

$$
\text { (unit) } x \Vdash 1_{\mathcal{S}}
$$

\footnotetext{
${ }^{4}$ Some early attempts to construct points in a formal topology can be found in [Valentini 93] but the lack of a suitable theory of subsets did not allow to obtain the completeness theorem.
} 
The second condition is that a point belongs to the intersection of two basic open sets if and only if it belongs to both, i.e. we require that for any point $x$ and for any $a, b \in S$

$$
\text { (intersection) } \quad x \Vdash a \bullet \mathcal{S} b \text { iff } x \Vdash a \text { and } x \Vdash b
$$

Finally we need to state that a point cannot be split by means of the basic open sets; to this aim we require that for any point $x$ and for any $a \in S$ and $U \subset S:$

$$
\text { (completeness) } \frac{x \Vdash a \quad a \triangleleft U}{(\exists u \varepsilon U) x \Vdash u}
$$

One can obtain the collection $\operatorname{Pt}(\mathcal{S})$ of formal points of the formal topology $\mathcal{S}$ by considering all the subsets $\alpha$ of $S$ which satisfy the following conditions for any $a, b \in S$ and for any $U \subset S$ :

$$
1_{\mathcal{S}} \varepsilon \alpha, \quad a \bullet_{\mathcal{S}} b \varepsilon \alpha \text { iff } a \varepsilon \alpha \text { and } b \varepsilon \alpha, \quad \frac{a \varepsilon \alpha \quad a \triangleleft U}{(\exists u \varepsilon U) u \varepsilon \alpha}
$$

since he can define the relation $\Vdash$ by putting

$$
\alpha \Vdash a \equiv a \varepsilon \alpha
$$

Let us came back to the prime filters of the lattice $\operatorname{Sat}(\mathcal{S})$. We can define a one-one correspondence $\phi$ between the collection of formal points $\operatorname{Pt}(\mathcal{S})$ and the collection of completely prime filters of $\operatorname{Sat}(\mathcal{S})$ by putting, for any $\alpha \in \operatorname{Pt}(\mathcal{S})$ :

$$
\phi(\alpha) \equiv\{[U]:(\exists u \varepsilon U) \alpha \Vdash u\},
$$

i.e. by associating to each (formal) point the completely prime filter of the open sets which contain it.

It should be clear now the reason why we are interested in the completely prime filters of the lattice $\operatorname{Sat}(\mathcal{S})$ but before one begins any search for them he should be aware that using a standard theory of subsets there is, in general, no hope to find enough completely prime filters. For instance, let us consider the formal topology defined over a complete atomless boolean algebra $\mathcal{H}^{5}$ by putting $a \triangleleft U \equiv a \leq \vee U$; in this case one can find no point at all in $\operatorname{Pt}(\mathcal{H})$ since points are just the completely prime filters of $\mathcal{H}$ and it is well known that there is a one-one correspondence between atoms and completely prime filters of $\mathcal{H}$ [Bell-Machover 77]. Of course the problem in this case is that a prime filter must respect an uncountable quantity of subsets in order to be a point; the way out we propose here is to change the theory of subsets in such a way that the number of subsets of a given set is at most countable so that a Rasiowa-Sikorski-like theorem be sufficient in order to obtain completely prime filters.

\footnotetext{
${ }^{5}$ For instance one can consider the regular open algebra of the closed unit interval $[0,1]$.
} 


\section{A Rasiowa-Sikorski-like theorem for countable lattices}

In this paragraph we will set up the technical device that we need in order to prove the completeness theorem. In particular we will show how any countable lattice with 0 and $1, \mathcal{L} \equiv\langle L, 0,1, \vee, \wedge\rangle$, can be embedded into a point-set topology in such a way that a countable quantity of suprema are respected, provided that $\wedge$ is distributive over such suprema. We must here stress on the fact that the proof we are going to show is classical and hence it is not possible to carry it on within ITT; for instance in case the lattice we are considering is countable we will show a theorem of existence of prime filters.

Let us begin by recalling some standard definitions. An order relation $\leq$ can be defined between two elements $x$ and $y$ of $L$ by putting $x \leq y \equiv x=x \wedge y$, then the following definitions make sense.

Definition 3.1 (Filter) Let $\mathcal{L}$ be a lattice. Then a subset $F$ of $L$ is a filter if:

$$
1 \varepsilon_{\mathcal{L}} F, \quad \frac{x \varepsilon_{\mathcal{L}} F \quad x \leq y}{y \varepsilon_{\mathcal{L}} F}, \quad \frac{x \varepsilon_{\mathcal{L}} F \quad y \varepsilon_{\mathcal{L}} F}{x \wedge y \varepsilon_{\mathcal{L}} F}
$$

In this paper we will be interested only in proper filters of $\mathcal{L}$, i.e. filters which are proper subsets of $L$ or, equivalently, which do not contain 0 .

Definition 3.2 (Prime filter) Let $\mathcal{L}$ be a lattice and $F$ one of its proper filters. Then $F$ is a prime filter of $\mathcal{L}$ if whenever $x \vee y \varepsilon_{\mathcal{L}} F$ then $x \varepsilon_{\mathcal{L}} F$ or $y \varepsilon_{\mathcal{L}} F$.

On account of what we said in the previous section, we are interested in a particular collection of prime filters, i.e. those which respect a countable quantity of subsets of $L$.

Definition 3.3 Let $\mathcal{L}$ be a lattice, $F$ one of its filters and $T$ a subset of $L$ which has a supremum in $\mathcal{L}$. Then $F$ respects $T$ if whenever $\vee T \varepsilon_{\mathcal{L}} F$ there exists b $\varepsilon_{\mathcal{L}} T$ such that $b \varepsilon_{\mathcal{L}} F$.

The main problem we have to solve now is to show that there exist prime filters which respect a countable quantity of subsets $T_{1}, \ldots, T_{n}, \ldots$ To this purpose we can prove the following theorem ${ }^{6}$ which guarantees the existence in any distributive lattice of a proper filter (not necessarily a prime filter) which respects $T_{1}, \ldots, T_{n}, \ldots$.

Theorem 3.4 Let $\mathcal{L}$ be a lattice, $x, y$ two elements of $L$ such that $x \not \leq y$ and $T_{1}, \ldots, T_{n}, \ldots$ a countable quantity of subsets of $L$ which have a supremum in $L$ such that $\wedge$ is distributive over them. Then there exists a filter $F$ of $\mathcal{L}$ which contains $x$, does not contain $y$ and respects all of the subsets $T_{1}, \ldots, T_{n}, \ldots$.

\footnotetext{
${ }^{6} \mathrm{~A}$ similar result, even if bounded to the case of complete boolean algebras, can be found in [Feferman 52], where it is called Tarski's lemma, but the proof we give here is different and more general of that of Tarski because the structures we are considering are weaker. Moreover J. Bell, after reading this paper, pointed out to me another related result for the problem of finding prime ideals in a countable Heyting algebra in [Horn 69].
} 
Proof. We can classically construct such a filter in a countable number of steps, starting from the filter ${ }^{7} F_{0}=\uparrow x \equiv\{z \in L: x \leq z\}$ and going on by extending it to a filter which respects all of the subsets $T_{1}, \ldots, T_{n}, \ldots$. Let us first construct a new countable list $W_{1}, \ldots, W_{m}, \ldots$ of subsets of $S$ out of the list $T_{1}, \ldots, T_{n}, \ldots$ in such a way that any subset $T_{i}$ appears a countable number of times among $W_{1}, \ldots, W_{m}, \ldots$. Now put $c_{0}=x$, and hence $F_{0}=\uparrow c_{0}$, and suppose, by inductive hypothesis, that we have constructed an element $c_{n}$ such that $c_{n} \not \leq y$ and we have defined $F_{n}=\uparrow c_{n}$, then put

$$
c_{n+1}=\left\{\begin{array}{cl}
c_{n} & \text { if } \vee W_{n} \not \mathcal{L} F_{n} \\
c_{n} \wedge b_{n} & \text { if } \vee W_{n} \varepsilon_{\mathcal{L}} F_{n}
\end{array}\right.
$$

where $b_{n}$ is an element of $W_{n}$ such that $c_{n} \wedge b_{n} \not \leq y$; in fact such an element exists because in the latter case $\vee W_{n} \varepsilon_{\mathcal{L}} F_{n}=\uparrow c_{n}$, and hence $c_{n} \leq \vee W_{n}$, then if for all $b \varepsilon_{\mathcal{L}} W_{n}, c_{n} \wedge b \leq y$ then, because of distributivity, $c_{n}=c_{n} \wedge \vee W_{n}=$ $\vee_{b \varepsilon_{\mathcal{L}} W_{n}} c_{n} \wedge b \leq y$ which is contrary to the inductive hypothesis.

Now we can define the filter $F_{n+1}=\uparrow c_{n+1}$ and it is immediate to check that $F_{n} \subseteq F_{n+1}$ and that $y \not \notin_{\mathcal{L}} F_{n+1}$ since $c_{n+1} \not \leq y$. Finally we put $F=\cup_{i \in \omega} F_{i}$. Then $F$ is a filter since it is the union of a chain of filters; moreover $x \varepsilon_{\mathcal{L}} F$ since $x \varepsilon_{\mathcal{L}} F_{0} \subseteq F$ while $y \Varangle_{\mathcal{L}} F=\cup_{i \in \omega} F_{i}$ otherwise there would be an $i \in \omega$ such that $y \varepsilon_{\mathcal{L}} F_{i}$ which is contrary to the way we have constructed the filters; moreover $F$ respects all of the subsets $T_{1}, \ldots, T_{n}, \ldots$ since if $\vee T_{n} \varepsilon_{\mathcal{L}} F=\cup_{i \in \omega} F_{i}$ then there is an $i \in \omega$ such that $\vee T_{n} \varepsilon_{\mathcal{L}} F_{i}$ and hence, since any $T_{n}$ appears a countable number of times in the list $W_{1}, \ldots, W_{m}, \ldots$, for some $h \geq i$ it happens that $W_{h}=T_{n}$ and hence $\vee W_{h}=\vee T_{n} \varepsilon_{\mathcal{L}} F_{i} \subseteq F_{h}$ and so there exists $b_{h} \varepsilon_{\mathcal{L}} T_{n}$ such that $b_{h} \varepsilon_{\mathcal{L}} F_{h+1} \subseteq F .8$

It should be noted that the theorem ensures the existence of a proper filter which respects a countable quantity of suprema without any condition at all, since $1 \not \leq 0$ always holds and 1 is contained in any filter.

The previous theorem can be used to construct a prime filter in case we are dealing with a countable distributive lattice.

Corollary 3.5 Let $\mathcal{L}$ be a countable distributive lattice, $x$, y two elements of $L$ such that $x \not \leq y$ and $T_{1}, \ldots, T_{n}, \ldots$ a countable quantity of subsets of $L$ which have a supremum in $L$ such that $\wedge$ is distributive over them. Then there exists a prime filter which contains $x$, does not contain $y$ and respects all of the subsets $T_{1}, \ldots, T_{n}, \ldots$

Proof. We have only to observe that in this case there is a countable quantity of binary suprema and hence we can use the previous theorem in order to obtain a filter which respects the countable quantity of binary suprema and the countable quantity of subsets $T_{1}, \ldots, T_{n}, \ldots$ It is then obvious that this filter is prime because it respects all the binary suprema.

\footnotetext{
${ }^{7}$ It is easy to check that $F_{0}$ is indeed a filter of $\mathcal{L}$.

${ }^{8}$ As the referee noted, the same proof works also for a slightly more general statement, i.e. the case $\mathcal{L}$ is a $\wedge$-semilattice instead of a lattice, but we think that the version given here better fit with the contents of the paper.
} 
The existence of prime filters which respect a given countable quantity of suprema is the key point to construct a point-set topology in which a countable distributive lattice $\mathcal{L}$ can be embedded using a morphism which respects the countable quantity of suprema of the subsets $T_{1}, \ldots, T_{n}, \ldots$ In fact, let us consider the collection $\operatorname{Pf}(\mathcal{L})=\{P$ : $P$ prime filter of $\mathcal{L}$ which respects $\left.T_{1}, \ldots, T_{n}, \ldots\right\}$ and any topology $\tau$ on $\operatorname{Pf}(\mathcal{L})$ such that the map defined by putting $\operatorname{ext}(x)=\left\{P \in P f(\mathcal{L}): x \varepsilon_{\mathcal{L}} P\right\}$ associates an open set of the topology $\tau$ to any element of $L$. It is immediate to see that the map ext respects all the finitary operations:

$$
\begin{aligned}
\operatorname{ext}(0) & =\left\{P \in \operatorname{Pf}(\mathcal{L}): 0 \varepsilon_{\mathcal{L}} P\right\}=\emptyset \\
\operatorname{ext}(1) & =\left\{P \in \operatorname{Pf}(\mathcal{L}): 1 \varepsilon_{\mathcal{L}} P\right\}=\operatorname{Pf}(\mathcal{L}) \\
\operatorname{ext}(x \vee y) & =\left\{P \in \operatorname{Pf}(\mathcal{L}): x \vee y \varepsilon_{\mathcal{L}} P\right\} \\
& =\left\{P \in \operatorname{Pf}(\mathcal{L}): x \varepsilon_{\mathcal{L}} P\right\} \cup\left\{P \in P f(\mathcal{L}): y \varepsilon_{\mathcal{L}} P\right\}=\operatorname{ext}(x) \cup \operatorname{ext}(y)
\end{aligned}
$$

since $x \vee y$ is contained in a prime filter $P$ if and only if $x \varepsilon_{\mathcal{L}} P$ or $y \varepsilon_{\mathcal{L}} P$, and

$$
\begin{aligned}
\operatorname{ext}(x \wedge y) & =\left\{P \in P f(\mathcal{L}): x \wedge y \varepsilon_{\mathcal{L}} P\right\} \\
& =\left\{P \in P f(\mathcal{L}): x \varepsilon_{\mathcal{L}} P\right\} \cap\left\{P \in \operatorname{Pf}(\mathcal{L}): y \varepsilon_{\mathcal{L}} P\right\}=\operatorname{ext}(x) \cap \operatorname{ext}(y)
\end{aligned}
$$

since $x \wedge y$ is contained in a filter $P$ if and only if $x \varepsilon_{\mathcal{L}} P$ and $y \varepsilon_{\mathcal{L}} P$.

Moreover ext respects all the suprema of the subsets $T_{1}, \ldots, T_{n}, \ldots$ that we are considering. In fact

$$
\begin{aligned}
\operatorname{ext}\left(\vee_{b \varepsilon_{\mathcal{L}} T_{i}} b\right) & =\left\{P \in P f(\mathcal{L}): \vee_{b \varepsilon_{\mathcal{L}} T_{T}} b \varepsilon_{\mathcal{L}} P\right\} \\
& =\cup_{b \varepsilon_{\mathcal{L}} T_{i}}\left\{P \in P f(\mathcal{L}): b \varepsilon_{\mathcal{L}} P\right\}=\cup_{b \in T_{i}} \operatorname{ext}(b)
\end{aligned}
$$

since $\vee_{b \varepsilon_{\mathcal{L}} T_{i}} b$ is contained in a prime filter $P$ which respects all of the sets $T_{1}, \ldots, T_{n}, \ldots$ if and only if there is an element $b \varepsilon_{\mathcal{L}} T_{i}$ such that $b \varepsilon_{\mathcal{L}} P$.

Finally we note that also the order relation is respected since for any couple of elements $x, y \in L$, if $x \leq y$ then $\operatorname{ext}(x) \subseteq \operatorname{ext}(y)$.

To conclude the proof that ext is an embedding of $\mathcal{L}$ into the topology $\tau$ we have to show that it is injective, i.e. that if $x \neq y$ then $\operatorname{ext}(x) \neq \operatorname{ext}(y)$. But this is an immediate consequence of 3.5 since if $x \neq y$ then $x \not \leq y$ or $y \not \leq x$ must hold and hence we can construct a prime filter $P$ which respects all of the considered suprema and "separates" $x$ and $y$, i.e. $P$ is an element which belongs to only one of the two sets $\operatorname{ext}(x)$ and $\operatorname{ext}(y)$.

The easiest way to define a topology on $\operatorname{Pf}(\mathcal{L})$ such that $\operatorname{ext}(x)$ is an open subset for each $x \in L$ is to consider the topology $\tau_{\mathcal{L}}$ whose base is any family of subsets $\{\operatorname{ext}(x): x \in \mathcal{B}\}$, where $\mathcal{B}$ is a base for the lattice $\mathcal{L}$ (see [Battilotti-Sambin 94]), i.e. a subset of $L$, closed under $\wedge$, such that any element in $L$ is the supremum of elements in $\mathcal{B}$. ${ }^{9}$ In fact, if $\mathcal{L}$ is a countable distributive lattice, we can force the prime filters in $\operatorname{Pf}(\mathcal{L})$ to respect all of the countable quantity of subsets of $\mathcal{B}$ which are needed in order to construct all the elements in $L$. Then, given $x \in L$ we know that $x=\vee\left\{b_{1}, \ldots, b_{n}, \ldots\right\}$ for a suitable choice of the elements $b_{1}, \ldots, b_{n}, \ldots \in \mathcal{B}$

\footnotetext{
${ }^{9}$ Note that any lattice $\mathcal{L}$ has at least one base, i.e. the collection $L$ itself. The deep reason of this definition is that the base $\mathcal{B}$ for $\mathcal{L}$ may well be a ITT set even if $L$ is only a collection.
} 
and hence $\operatorname{ext}(x)=\operatorname{ext}\left(\vee\left\{b_{1}, \ldots, b_{n}, \ldots\right\}\right)=\cup_{b \varepsilon\left\{b_{1}, \ldots, b_{n}, \ldots\right\}} \operatorname{ext}(b) \in \tau$. In the following we will call the topology $\tau_{\mathcal{L}}$ the point-set topology associated to $\mathcal{L}$.

\section{The completeness theorem}

Now we are going to apply the algebraic results of the previous section to show the completeness theorem for the formal topologies. Let us first give a formal definition of what a valuation of a formal topology into a point-set topology is.

Definition 4.1 (Valuation) Let $\mathcal{S}$ be a formal topology and $\tau=\langle X, \Omega(X)\rangle$ be a point-set topology. Then a valuation $V$ of the formal topology $\mathcal{S}$ into $\tau$ is a map of the elements of $S$ into the open subsets of $\tau$ such that $V\left(1_{\mathcal{S}}\right)=X$ and $V(a \bullet \mathcal{S} b)=V(a) \cap V(b)$ which is extended to a subset $U$ of $S$ by putting $V(U)=\cup_{u \varepsilon U} V(u)$.

This definition allows to state in a mathematical way what, in the second section, had been called the intended meaning of the cover relation. In fact it is immediate to check that all the conditions we required on a cover relation are valid under any valuation $V$ provided that $a \triangleleft_{\mathcal{S}} U$ is interpreted as $V(a) \subseteq$ $V(U)$. Hence the following validity theorem holds.

Theorem 4.2 (Validity) Let $\mathcal{S}$ be a formal topology, $\tau$ a point-set topology and $V$ a valuation of $\mathcal{S}$ into $\tau$. Then if $U \triangleleft_{\mathcal{S}} W$ then $V(U) \subseteq V(W)$.

Our aim is to show that also the other implication holds, that is we want to prove the following theorem.

Theorem 4.3 (Completeness) Let $\mathcal{S}$ be a formal topology, and suppose that for any point-set topology $\tau$ and for any valuation $V$ of $\mathcal{S}$ into $\tau, V(U) \subseteq V(W)$ holds. Then $U \triangleleft_{\mathcal{S}} W$.

In order to prove this theorem, we will show that there exists a suitable topology $\tau^{*}$ and a suitable valuation $V^{*}$ of $\mathcal{S}$ into $\tau^{*}$ such that if $U \oiint_{\mathcal{S}} W$ then $V^{*}(U) \nsubseteq V^{*}(W)$, which is classically equivalent. The key point to find such a topology is to consider the topology $\tau_{O p e n(\mathcal{S})}$ associated to the countable distributive lattice $O p e n(\mathcal{S})$ (provided that we can construct it!).

The first step is then to find a base for the lattice $\operatorname{Open}(\mathcal{S})$ but this is an easy task since one can see that the equivalence classes $\{[\{a\}]: a \in S\}$ form a base for the lattice $\operatorname{Open}(\mathcal{S})$ since, for any subset $U$ of $S,[U]=\vee_{u \varepsilon_{\mathcal{S}} U}[\{u\}]$.

The second step is to observe that, as we work within an intuitionistic countable theory of sets and subsets, not only the distributive lattice $\operatorname{Open}(\mathcal{S})$ but also the number of suprema we can construct on its elements is countable since they are at most as many as the sets of ITT which can be used as index-sets are and the sets of ITT are a countable collection since they are exactly ${ }^{10}$

\footnotetext{
${ }^{10}$ With respect to this point the status of ITT is unlike a standard set theory which is only a syntax to describe some intended external model which is in general not completely specifiable so that the fact that the language is countable does not guarantee that the number of sets in the model is countable.
} 
what one can form using the ITT formation rules [Martin-Löf 84]. Hence all the results of the previous section apply, i.e. we can construct the point-set topology $\tau_{\text {Open }(\mathcal{S})}$, associated with the lattice $\operatorname{Open}(\mathcal{S})$, whose base are the subsets $a^{*} \equiv\left\{P \in \operatorname{Pf}(\operatorname{Open}(\mathcal{S})):[\{a\}] \varepsilon_{\operatorname{Open}(\mathcal{S})} P\right\}$, for any $a \in S$. Moreover we know that the lattice $\operatorname{Open}(\mathcal{S})$ can be embedded into $\tau_{\text {Open }}(\mathcal{S})$ using the map ext defined on the base of $\operatorname{Open}(\mathcal{S})$ by putting $\operatorname{ext}([\{a\}])=a^{*}$ and which respects all the countable quantity of existing suprema. So the topology $\tau_{\text {Open }(\mathcal{S})}$ is the point-set counterpart of the formal topology $\mathcal{S}$, i.e. it has the "same" base and the "same" open sets, the only novelty being that we have succeeded in filling the open sets of $\tau_{O p e n(\mathcal{S})}$ of points instead of dealing with purely formal objects.

These observations have finally led to the solution of our problem, i.e. we have found the valuation $V^{*}$ we were looking for, since we can define it by simply putting $V^{*}(a) \equiv a^{*}$. We can immediately verify that $V^{*}$ is indeed a valuation since $V^{*}\left(1_{\mathcal{S}}\right)=\left\{P \in \operatorname{Pf}(\operatorname{Open}(\mathcal{S})):\left[\left\{1_{\mathcal{S}}\right\}\right] \varepsilon_{\operatorname{Open}(\mathcal{S})} P\right\}=\operatorname{Pf}(\operatorname{Open}(\mathcal{S}))$ and $V^{*}(a \bullet \mathcal{S} b)=(a \bullet \mathcal{S} b)^{*}=\operatorname{ext}([\{a \bullet \mathcal{S} b\}])=\operatorname{ext}([\{a\}]) \cap \operatorname{ext}([\{b\}])=a^{*} \cap b^{*}=$ $V^{*}(a) \cap V^{*}(b)$. Moreover by using this definition we obtain that $V^{*}(U)=$ $\cup_{u \varepsilon U} V^{*}(u)=\cup_{u \varepsilon U} \operatorname{ext}([\{u\}])=\operatorname{ext}\left(\vee_{u \varepsilon U}[\{u\}]\right)=\operatorname{ext}\left(\left[\cup_{u \varepsilon U}\{u\}\right]\right)=\operatorname{ext}([U])$ and hence if $U \nexists_{\mathcal{S}} W$ then $[U] \operatorname{LOpen}(\mathcal{S})[W]$ and so $V^{*}(U)=\operatorname{ext}([U]) \nsubseteq$ $\operatorname{ext}([W])=V^{*}(W)$.

It is worth noting that the point-set topology $\tau_{O p e n(\mathcal{S})}$ and the valuation $V^{*}$ do not depend on the particular subsets $U$ and $V$ we are considering but only on the formal topology $\mathcal{S}$, i.e. we have proved a result which is stronger than what we need: the point-set topology $\tau_{\text {Open }(\mathcal{S})}$ and the valuation $V^{*}$ provide us with a canonical model of the formal topology $\mathcal{S}$.

\section{Conclusions}

Some comments on the proof of the completeness theorem may be useful. As it stands, the proof is carried on using a classical meta-mathematics and hence it is not possible to formalize it within the framework of ITT, or within any other constructive one. It may be considered as the proof a classically minded mathematician can produce when "playing" with formal topology. Moreover the result deeply depends on the particular theory of subsets we adopt here to deal with formal topology: in particular it holds for any countable theory of sets with a countable theory of subsets. Hence, from a strictly constructive point of view the provided proof can only exclude that it is impossible to obtain a completeness theorem. Anyhow from a constructive perspective such a theorem is still missing and this classical proof is the best information we have.

Acknowledgments. I'm indebted to G. Sambin and the referee which, besides many useful observations on the contents of the paper and the way the topics are presented, pointed out an error in the proof of 3.4 in a preliminary version of this work. 


\section{References}

[Battilotti-Sambin 94] G. Battilotti, G. Sambin, Pretopologies and a uniform presentation of sup-lattices, quantales and frames, to appear.

[Bell-Machover 77] J.L. Bell, M. Machover, A course in Mathematical Logic, North Holland Publishing Company, 1977.

[Feferman 52] S. Feferman, Review of Rasiowa and Sikorski, The Journal of Symbolic Logic, vol 17 (1952), pp. 72.

[Fourman-Grayson 82] M.P .Fourman, R.J. Grayson, Formal Spaces, Proceedings of The L.E.J. Brouwer Centenary Symposium, eds. A.S. Troelstra and D. van Dalen, North Holland 1982, pp. 107-122.

[Horn 69] A. Horn, Logic with truth values in a linearly ordered Heyting algebra, The Journal of Symbolic Logic, vol 34, n. 3 (1969), pp. 395-408.

[Johnstone 82] P.T. Johnstone, Stone Spaces, Cambridge studies in advanced mathematics 3., Cambridge University Press, 1982 .

[Martin-Löf 84] P. Martin-Löf, Intuitionistic type theory, notes by Giovanni Sambin of a series of lectures given in Padua, June 1980, Bibliopolis, Naples,1984.

[Rasiowa-Sikorski 51] H. Rasiowa, R. Sikorski, A proof of the completeness theorem of Gödel, Fundamenta Mathematica 37, pp. 193200.

[Sambin 87]

G. Sambin, Intuitionistic formal spaces - a first communication, Mathematical logic and its applications, D. Skordev ed., Plenum, 1987, pp. 187-204.

[Sambin 88] G. Sambin, Intuitionistic formal spaces and their neighborhood, Proceedings of Logic Colloquium '88, R Ferro, C. Bonotto, S. Valentini, A. Zanardo ed., North-Holland, 1989, pp. 261-286.

[Sambin-Valentini 9?] G. Sambin, S. Valentini, Building up a tool-box for Martin-Löf intuitionistic type theory, to appear.

[Valentini 93] S. Valentini, Points and Co-Points in Formal Topology, Bollettino dell'Unione Matematica Italiana, 7-A, 1993, pp. 7-19. 